Afrah Kh Al-Hamdany BDS, MSc (Assist Prof)

Nagham H Kassab BDS, MSc (Lect)

\section{Correlation of Vertical Dimensions of Soft Tissue Facial Profiles}

\author{
Dept of Pedod, Orthod, and Prev Dentistry \\ College of Dentistry, University of Mosul
}

Department of Prosthetic Dentistry

College of Dentistry, University of Mosul

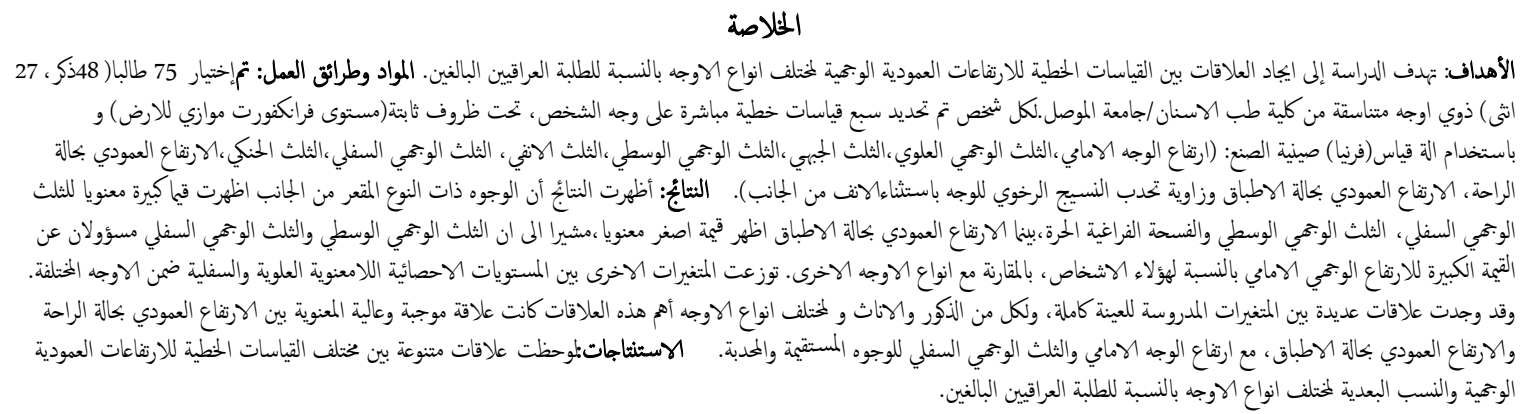

الخلاصة

\section{ABSTRACT}

Aims: To establish the interrelationships of linear measurements of the vertical facial heights among different facial profiles in young Iraqi adult students. Materials and Methods: 75 students (48 males, 27 females) of pleasing face, balanced facial profile were selected from the College of Dentistry, University of Mosul. For each subject seven vertical linear measurements \{ Anterior facial height (n-gn), upper facial third, frontal third $(\operatorname{tr}-\mathrm{n})$, middle facial third, nasal third $(\mathrm{n}-\mathrm{sn})$, lower facial third, gnathic third, (sn-gn), rest vertical dimension (RVD)(pn-pog), occlusal vertical dimension (OVD) (pn-pog) and angle of soft tissue profile facial convexity excluding the nose (n-sn-pog) $\}$ were measured directly on the subject's face under standarized conditions keeping Frankfort plane parallel to floor, using electronic digital vernier caliper (China). Results: sn-me, n-sn and free way space are significantly larger, while pn-pog (OVD) is significantly smaller in concave facial profiles $(p \leq 0.05)$ indicating that the lower and middle facial thirds are responsible for the greater anterior facial height in these subjects compared with other facial profiles' subjects. The remaining variables distributed on statistical levels of difference between the upper and lower levels with non-significant difference $(p>0.05)$ within facial profiles. Various correlations were noticed among all the studied variables for total sample, males and females and in different facial profiles. Of most important, is the positive significantly high correlation of pn-pog (OVD) and pn-pog (RVD) with n-me, sn-me for convex and straight profiles. Conclusions: From this study ,spatial relationships among various vertical facial dimensions and the dimensional proportions of different facial profiles in young Iraqi adult students were noticed.

Key Words: Esthetics, Facial profile, Occlusal vertical dimension, Rest vertical dimension.

Al-Hamdany AKh, Kassab NH. Correlation of Vertical Dimensions of Soft Tissue Facial Profiles. $\mathrm{Al}$

Rafidain Dent J. 2010; 10(2): 243-253.

Received: $17 / 2 / 2009$

Sent to Referees: $22 / 2 / 2009$

Accepted for Publication: 21/4/2009

\section{INTRODUCTION}

Esthetics is the primary reason why patients seek orthodontic/prosthodontic treatment and the resulting soft tissue profile is their measure of therapeutic success ${ }^{(1)}$. Attention to physical appearance, particularly of the face, has become a very important issue in modern society ${ }^{(2)}$. Although evi- 
dence exists for a universal standard for proportions involved in facial attractiveness ${ }^{(3)}$, there may be differences in perception of the soft tissue drape among ethnic groups ${ }^{(4)}$.

The search for the profile with ideal proportions is one of the oldest aims of art. These ideal proportions provide the basic standard for assessment of the average profile-mean value, biometric mean, or average. The profile may be divided into three approximately equal parts: Frontal third (tr$\mathrm{n})$, nasal third (n-sn), gnathic third (sn-gn). The gnathic third may be up to a tenth greater rather than smaller ${ }^{(5)}$.

Similar proportion ( $\mathrm{n}-\mathrm{gn})$ with mid-face (n-sn) may be seen with respect to anterior face height, occupying $45 \%$, the lower face (sn-gn) $55 \%$ of the total height ${ }^{(5)}$.

Number of methods have been used to evaluate the facial esthetic (two dimensional) including anthropometry ${ }^{(6-9)}$, photogrammetry ${ }^{(9)}$, cephalometry ${ }^{(10-12)}$, and computer imaging $^{(13,14)}$. Current technology provides several noninvasive image analysis systems for indirect computerized facial anthropometry including stereophotogrammetry, laser scanning, range cameras, optoelectronic instruments, and electromagnetic digitizers $^{(15-20)}$. In addition, ultrasonography allows facial anthropometry to be performed even during intrauterine life ${ }^{(21,22)}$.

These instruments provide the threedimensional coordinates of selected landmarks, and euclidean geometric calculations can be used to obtain three-dimensional linear distances of selected facial structures, as well as facial areas and volumes ${ }^{(15-23)}$.

Both of vertical dimensions, the occlusal (OVD) and the rest vertical (RVD) dimensions are subjected to change resulting from loss of teeth ${ }^{(24)}$, orthodontic and/or orthopedic treatment ${ }^{(25)}$. The physiologic rest position has been considered by many authors to remain constant throughout life regardless the presence or absence of teeth ${ }^{(26)}$.

Although there appears to be considerable agreement across cultures about what facial anatomical relationships are attractive, there are variations in the soft-tissue drape related to possible cultural influences on the perception of attractiveness ${ }^{(27)}$.
An important point to be considered is that this study concentrated only on pleasing faces individuals, malocclusions were omitted from study group. According to Kim et $a l .,{ }^{(28)}$ the range of a normal occlusion includes quite diverse anteroposterior and vertical skeletal relationships.

The purpose of the current study was to establish the linear measurements of the vertical facial heights among different facial profiles in young Iraqi adult students, to report the presence of any sexual dimorphism in these parameters and to demonstrate the interrelationships among these parameters.

\section{MATERIALS AND METHODS}

The sample consists of 75 students (48 males, 27 females) satisfying the criteria of pleasing face, balanced facial profile, competent lips, and normal overjet and overbite relationship, were selected independently by two investigators from the College of Dentistry/University of Mosul. Subjects who satisfied these criteria even when they had malocclusions were not excluded from the study ${ }^{(29)}$. The subjects were randomly selected healthy subjects, of estimated mean age 21 years and five months and with the permanent dentition completely erupted (except for wisdom teeth). The subjects had no congenital anomalies and no significant facial, dental asymmetries, none of these subjects had undergone, orthopedic, orthodontic treatment or, orthognathic surgery and no previous history to facial trauma. Each subject was seated on a dental chair, asked about name, medical and dental history.

Extra and intra oral clinical assessment had been made, A pin-head sized marks were marked by an indelible pencil on the skin before measurement using water soluble marker.

Seven vertical linear measurements were measured directly on the subject's face and taken under standardized conditions keeping Frankfort plane parallel to floor. During registration of the rest vertical dimension (RVD), the subject's head must be erect, teeth separated and the lips gently touch with masticatory muscles completely 
relaxed $^{(30)}$. The occlusal vertical dimension (OVD) was determined by asking subject to relax and occlude his teeth in centric occlusion with lips closed, and the distance between the two marks was measured. The electronic digital vernier caliper (Lezaco Art 2771, 0-150 mm, 0-6 inch accuracy, China) was used to measure the seven vertical linear measurements in millimeters.

To make landmark determination as consistent as possible, a given landmark was identified for each subject at one sitting. Each was then checked by another investigator. In order to minimize measurement error, all linear measurements were performed by two investigators working independently. Intra-investigator and inter-investigator measurement error was predetermined at 0.5 $\mathrm{mm}$, and no significant difference were no- ticed between the two measurements at $p \leq$ 0.05 .

The following soft tissue landmarks were defined as showed in Figure (1): Trichion (tr): A point located at the hairline of the forehead ${ }^{(5,6,9)}$, soft tissue nasion (n): The most posterior point on the tissue overlying the frontonasal suture ${ }^{(29,31)}$, pronasale (pn): The most prominent or anterior point of the nose ${ }^{(29,31)}$, subnasale (sn): The point at which the columella merges with the upper coetaneous $\operatorname{lip}^{(8,9,29,31)}$, soft tissue b-point (b): The point of greatest concavity in the midline of the lower lip between lower milion border and soft tissue pogonion $^{(8,9,29,31)}$, soft tissue pogonion (pog): The most anterior point of the soft tissue $\operatorname{chin}^{(29,31)}$, soft tissue menton (me): The most inferior point of the soft tissue chin ${ }^{(29,31)}$.

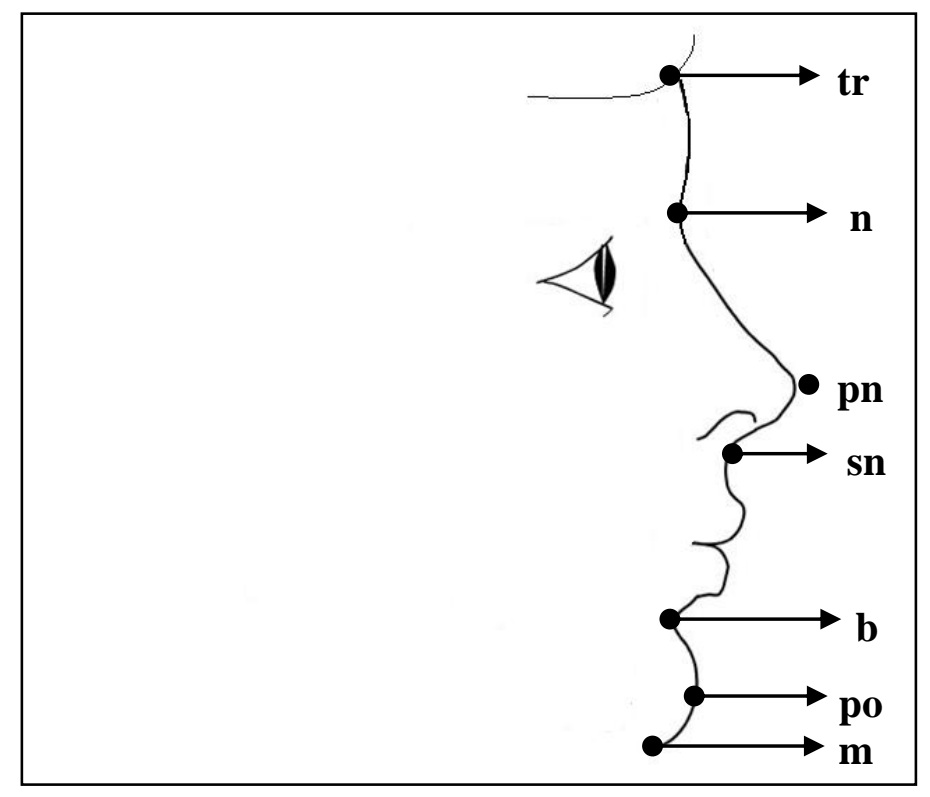

Figure(1): Soft tissue landmarks. 
While soft tissue measurements included the following:

1. Anterior facial height ( $\mathrm{n}-\mathrm{gn})$ : the distance between point $(\mathrm{n})$ and point $(\mathrm{gn})^{(5)}$.

2. Upper facial third, frontal third $(\operatorname{tr}-\mathrm{n})$ : the distance between point $t r$ and point $n^{(5)}$.

3. Middle facial third, nasal third $(n-s n)$ : the distance between point $\mathrm{n}$ and point $\mathrm{sn}^{(5)}$.

4. Lower facial third, gnathic third (sn-gn): the distance between point sn and point $\mathrm{gn}^{(5)}$.

5. Rest vertical dimension (RVD)(pn-pog): the postural position of the mandible when an individual is resting comfortably in upright position and associated muscles are in state of minimal contractual activity $^{(30,32)}$.

6. Occlusal vertical dimension (OVD) (pnpog): the distance measured between two points when occluding member are in con$\operatorname{tact}^{(30,32)}$.

7. Angle of soft tissue profile facial convexity excluding the nose $(n-s n-p o g)^{(31,33)}$.

For statistical analysis descriptive sta- tistics including the mean, standard deviation, were calculated. Student $t$ test was used to show the statistical difference between males and females. ANOVA and Duncan's multiple analysis range tests are used to reveal the statistical difference among different facial profiles. Pearson correlation coefficient was carried out among all the variables for males, females and total sample and in different facial profiles separately. Correlation is considered significant at $p \leq 0.05$ level and highly significant at $p \leq 0.01$ level.

\section{RESULTS}

The descriptive statistics including mean and standard deviations of all variables for the total sample, both males and females are presented in Table (1) which shows significant differences between males and females for all the studied variables with the males having the greater values at $p \leq 0.05$.

Table (1): Descriptive statistics of all variables for the total sample, males and females.

\begin{tabular}{ccccccccc}
\hline \multirow{2}{*}{ Variable } & \multicolumn{2}{c}{ Total=75 } & \multicolumn{2}{c}{ Males=48 } & \multicolumn{2}{c}{ Females=27 } & \multirow{2}{*}{ t-value } & \multirow{2}{*}{ Sig.** } \\
& mean & SD & mean & SD & Mean & SD & & \\
\hline $\begin{array}{c}\text { Soft tissue facial } \\
\text { convexity* }\end{array}$ & 2.329 & 0.619 & 2.286 & 0.677 & 2.407 & 0.501 & $3.017-$ & $\mathrm{S}$ \\
\hline $\mathbf{n}-\mathbf{m e}$ & 120.060 & 8.564 & 123.962 & 7.275 & 112.978 & 5.750 & 7.692 & $\mathrm{~S}$ \\
\hline Tr-n & 62.698 & 8.239 & 64.757 & 7.647 & 58.962 & 8.085 & 1.951 & $\mathrm{~S}$ \\
\hline $\mathbf{n}-\mathbf{s n}$ & 56.023 & 4.026 & 57.411 & 3.805 & 53.506 & 3.133 & 3.447 & $\mathrm{~S}$ \\
\hline sn-me & 66.709 & 6.965 & 68.953 & 6.834 & 62.637 & 5.199 & 6.190 & $\mathrm{~S}$ \\
\hline pn-pog(OVD) & 66.736 & 6.468 & 68.247 & 6.134 & 63.993 & 6.254 & 3.309 & $\mathrm{~S}$ \\
\hline pn-pog(RVD) & 68.974 & 6.427 & 70.659 & 6.233 & 65.914 & 5.689 & 4.050 & $\mathrm{~S}$ \\
\hline Free way space & 2.5470 & 5.4031 & 3.2850 & 5.132 & 2.3280 & 2.9099 & 2.075 & $\mathrm{~S}$ \\
\hline
\end{tabular}

*Facial convexity: $0=$ concave, $1=$ straight, $2=$ slightly convex, $3=$ convex; $* * S=$ significant at $p \leq 0.05$; OVD=occlusal vertical dimension, RVD=rest vertical dimension.

The one way analysis of variance (ANOVA) comparing different facial profiles revealing significant differences among the three facial profiles for $n-s n$ and $\mathrm{sn}-\mathrm{me}$ and free way space (at $p \leq 0.05$ ), Table (2).

The results. of Duncan's multiple analysis range test (Table 3) showed that sn-me, n- sn and free way space are significantly larger, while pn-pog (OVD) is significantly smaller in concave facial profiles $(p \leq$ 0.05 ). The remaining variables distributed on statistical levels of difference between the upper and lower levels with non- significant difference $(p \leq 0.05)$ within facial profiles. 
Table (2): Analysis of Variance (ANOVA) comparing different facial profiles.

\begin{tabular}{|c|c|c|c|c|c|c|}
\hline Variable & & Sum of Squares & df & $\begin{array}{c}\text { Mean } \\
\text { Square }\end{array}$ & $\mathbf{F}$ & Sig. \\
\hline \multirow{3}{*}{$\begin{array}{c}\text { Soft tissue facial } \\
\text { convexity* }\end{array}$} & Between Groups & 56.022 & 3 & \multirow{3}{*}{$\begin{array}{c}18.674 \\
1.358\end{array}$} & \multirow{3}{*}{1374.969} & \multirow{3}{*}{.000} \\
\hline & Within Groups & .964 & 71 & & & \\
\hline & Total & 56.987 & 74 & & & \\
\hline \multirow[b]{3}{*}{ n-me } & Between Groups & 333.790 & 3 & \multirow{3}{*}{$\begin{array}{c}111.263 \\
66.344\end{array}$} & \multirow{3}{*}{1.677} & \multirow{3}{*}{.180} \\
\hline & Within Groups & 4710.423 & 71 & & & \\
\hline & Total & 5044.213 & 74 & & & \\
\hline \multirow{3}{*}{ tr-n } & Between Groups & 33.388 & 3 & & \multirow{3}{*}{.154} & \multirow{3}{*}{.927} \\
\hline & Within Groups & 5127.871 & 71 & 11.129 & & \\
\hline & Total & 5161.259 & 74 & & & \\
\hline \multirow[b]{3}{*}{ n-sn } & Between Groups & 226.412 & 3 & \multirow{3}{*}{$\begin{array}{l}75.471 \\
14.362\end{array}$} & \multirow{3}{*}{5.255} & \multirow{3}{*}{.002} \\
\hline & Within Groups & 1019.706 & 71 & & & \\
\hline & Total & 1246.118 & 74 & & & \\
\hline \multirow{3}{*}{ sn-me } & Between Groups & 264.155 & 3 & \multirow{3}{*}{$\begin{array}{l}88.052 \\
43.626\end{array}$} & \multirow{3}{*}{2.018} & \multirow{3}{*}{.119} \\
\hline & Within Groups & 3097.446 & 71 & & & \\
\hline & Total & 3361.601 & 74 & & & \\
\hline \multirow{3}{*}{ pn-pog(OVD) } & Between Groups & 181.085 & 3 & \multirow{3}{*}{$\begin{array}{l}60.362 \\
37.320\end{array}$} & \multirow{3}{*}{1.617} & \multirow{3}{*}{.193} \\
\hline & Within Groups & 2649.725 & 71 & & & \\
\hline & Total & 2830.811 & 74 & & & \\
\hline \multirow{3}{*}{ pn-pog(RVD) } & Between Groups & 121.612 & 3 & \multirow{3}{*}{$\begin{array}{l}40.537 \\
37.349\end{array}$} & \multirow{3}{*}{1.085} & \multirow{3}{*}{.361} \\
\hline & Within Groups & 2651.744 & 71 & & & \\
\hline & Total & 2773.356 & 74 & & & \\
\hline \multirow{3}{*}{ Free way space } & Between Groups & 54.724 & 3 & \multirow{3}{*}{$\begin{array}{l}18.241 \\
3.707\end{array}$} & \multirow{3}{*}{4.921} & \multirow{3}{*}{.004} \\
\hline & Within Groups & 263.198 & 71 & & & \\
\hline & Total & 317.922 & 74 & & & \\
\hline
\end{tabular}

*Facial convexity: $0=$ concave, $1=$ straight, $2=$ slightly convex, $3=$ convex; **Tabulated $\mathrm{F}=2.6802$ at $p \leq 0.05$; $\mathrm{OVD}=$ occlusal vertical dimension; $\mathrm{RVD}=$ rest vertical dimension. 
Table (3): Descriptive statistics with Duncan's multiple analysis range test comparing different facial profiles.

\begin{tabular}{|c|c|c|c|c|c|c|c|c|}
\hline Variable & Facial profiles & No. & Mean & SD & SE & Minimum & Maximum & $\begin{array}{l}\text { Duncan's } \\
\text { groups } * *\end{array}$ \\
\hline \multirow{5}{*}{$\begin{array}{l}\text { Soft tissue } \\
\text { facial con- } \\
\text { vexity* }\end{array}$} & Concave & 2 & .0000 & .0000 & .0000 & .00 & .00 & \multirow{5}{*}{$\begin{array}{l}\text { A } \\
\text { B } \\
\text { B } \\
\text { B }\end{array}$} \\
\hline & Straight & 22 & 1.0000 & .0000 & .0000 & 1.00 & 1.00 & \\
\hline & Slightly convex & 23 & 2.0000 & .0000 & .0000 & 2.00 & 2.00 & \\
\hline & Convex & 28 & 2.9643 & .1890 & 3.571 & 2.00 & 3.00 & \\
\hline & Total & 75 & 2.0133 & .8775 & .1013 & .00 & 3.00 & \\
\hline \multirow{5}{*}{ n-me } & Concave & 2 & 131.8750 & 1.5910 & 1.1250 & 130.75 & 133.00 & $\mathrm{~A}$ \\
\hline & Straight & 22 & 119.4309 & 9.7592 & 2.0807 & 105.56 & 136.85 & B \\
\hline & Slightly convex & 23 & 121.8513 & 6.5267 & 1.3609 & 110.98 & 136.60 & B \\
\hline & Convex & 28 & 119.8950 & 8.0982 & 1.5304 & 105.45 & 134.31 & B \\
\hline & Total & 75 & 120.6783 & 8.2562 & .9533 & 105.45 & 136.85 & \\
\hline \multirow{5}{*}{$\operatorname{tr}-\mathbf{n}$} & Concave & 2 & 63.1750 & 1.1667 & .8250 & 62.35 & 64.00 & \multirow{5}{*}{$\begin{array}{l}\text { A } \\
\text { A } \\
\text { A } \\
\text { A }\end{array}$} \\
\hline & Straight & 22 & 61.5095 & 10.1599 & 2.1661 & 39.37 & 80.41 & \\
\hline & Slightly convex & 23 & 63.1974 & 8.2694 & 1.7243 & 50.99 & 78.95 & \\
\hline & Convex & 28 & 62.4893 & 7.3394 & 1.3870 & 41.93 & 76.01 & \\
\hline & Total & 75 & 62.4373 & 8.3515 & .9643 & 39.37 & 80.41 & \\
\hline \multirow{5}{*}{ n-sn } & Concave & 2 & 66.0800 & 1.3011 & .9200 & 65.16 & 67.00 & \multirow{5}{*}{$\begin{array}{l}\text { A } \\
\text { B } \\
\text { B } \\
\text { B }\end{array}$} \\
\hline & Straight & 22 & 55.1541 & 4.4242 & .9433 & 46.84 & 62.01 & \\
\hline & Slightly convex & 23 & 55.9817 & 2.8873 & .6020 & 51.18 & 64.31 & \\
\hline & Convex & 28 & 56.6768 & 3.9607 & .7485 & 48.90 & 65.03 & \\
\hline & Total & 75 & 56.2677 & 4.1036 & .4738 & 46.84 & 67.00 & \\
\hline \multirow{5}{*}{ sn-me } & Concave & 2 & 65.3500 & .9192 & .6500 & 64.70 & 66.00 & \multirow{5}{*}{$\begin{array}{l}\text { A } \\
\text { A } \\
\text { A } \\
\text { A }\end{array}$} \\
\hline & Straight & 22 & 64.7650 & 6.9795 & 1.4880 & 54.55 & 80.67 & \\
\hline & Slightly convex & 23 & 69.5496 & 6.0486 & 1.2612 & 55.54 & 79.81 & \\
\hline & Convex & 28 & 67.2136 & 6.8550 & 1.2955 & 54.20 & 80.87 & \\
\hline & Total & 75 & 67.1620 & 6.7400 & .7783 & 54.20 & 80.87 & \\
\hline \multirow{5}{*}{$\begin{array}{c}\text { pn- } \\
\operatorname{pog}(\text { OVD })\end{array}$} & Concave & 2 & 59.1200 & 1.2445 & .8800 & 58.24 & 60.00 & \multirow{5}{*}{$\begin{array}{l}\text { A } \\
\text { B } \\
\text { B } \\
\text { B }\end{array}$} \\
\hline & Straight & 22 & 66.1395 & 6.7954 & 1.4488 & 55.33 & 76.55 & \\
\hline & Slightly convex & 23 & 68.0422 & 4.8720 & 1.0159 & 58.67 & 75.39 & \\
\hline & Convex & 28 & 67.7868 & 6.5440 & 1.2367 & 54.04 & 80.36 & \\
\hline & Total & 75 & 67.1508 & 6.1850 & .7142 & 54.04 & 80.36 & \\
\hline \multirow{5}{*}{$\begin{array}{c}\text { pn- } \\
\operatorname{pog}\left(R_{V D}\right)\end{array}$} & Concave & 2 & 64.4050 & 1.9870 & 1.4050 & 63.00 & 65.81 & \multirow{5}{*}{$\begin{array}{l}\text { A } \\
\text { A } \\
\text { A } \\
\text { A }\end{array}$} \\
\hline & Straight & 22 & 68.1543 & 6.7288 & 1.4346 & 56.67 & 78.43 & \\
\hline & Slightly convex & 23 & 70.5891 & 5.2927 & 1.1036 & 60.07 & 80.55 & \\
\hline & Convex & 28 & 69.8268 & 6.3266 & 1.1956 & 56.39 & 82.33 & \\
\hline & Total & 75 & 69.4254 & 6.1219 & .7069 & 56.39 & 82.33 & \\
\hline \multirow{5}{*}{$\begin{array}{l}\text { Free way } \\
\text { space }\end{array}$} & Concave & 2 & 7.2850 & .4031 & .2850 & 7.00 & 7.57 & A \\
\hline & Straight & 22 & 2.0150 & 2.1321 & .4546 & -4.89 & 4.80 & B \\
\hline & Slightly convex & 23 & 2.5470 & 9099 & .1897 & 1.40 & 5.16 & \multirow{3}{*}{$\begin{array}{l}\text { B } \\
B\end{array}$} \\
\hline & Convex & 28 & 2.0400 & 2.3520 & .4445 & -8.57 & 6.00 & \\
\hline & Total & 75 & 2.3280 & 2.0727 & .2393 & 8.57 & 7.57 & \\
\hline
\end{tabular}

*Facial convexity: $0=$ concave, $1=$ straight, $2=$ slightly convex, $3=$ convex; ** Different letters mean significant difference ( $\mathrm{p} \leq 0.05) ; \mathrm{OVD}=$ occlusal vertical dimension; $\mathrm{RVD}=$ rest vertical dimension. 
Table (4) describes the correlation coefficient among all the variables for males, females and total sample separately, of most important, is the positive highly significant correlation of pn-pog (OVD) and pn-pog (RVD with n-me, sn-me for males, females and total sample.

Also the positive significant correlation of free way space with $\mathrm{n}-\mathrm{sn}$ for total sample.

Table (4): The correlation coefficient among all the variables for males, females and total sample.

\begin{tabular}{|c|c|c|c|c|c|c|c|c|c|}
\hline Variable & Sex & 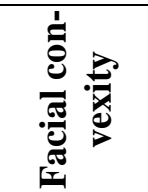 & $\underset{\Xi}{I}$ & $\stackrel{I}{I}$ & $\begin{array}{l}\text { कี } \\
\text { I }\end{array}$ & $\underset{\tilde{E}}{\stackrel{\Xi}{\Xi}}$ & $\pm \underset{\partial}{0}$ & 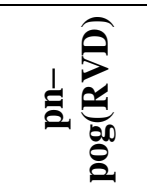 & 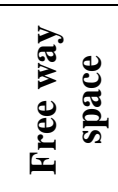 \\
\hline \multirow{2}{*}{$\begin{array}{c}\text { Soft tissue } \\
\text { facial convex- } \\
\text { ity }\end{array}$} & $\mathrm{T}$ & & & & & & & $0.356 * *$ & \\
\hline & $\begin{array}{l}\mathrm{M} \\
\mathrm{F}\end{array}$ & & & & & & & & \\
\hline \multirow{3}{*}{ n-me } & $\mathrm{T}$ & & & $0.345 * *$ & & $0.321 * *$ & $0.709 * *$ & $0.725 * *$ & \\
\hline & M & & & $0.688 * *$ & $0.571 * *$ & $0.282 *$ & $0.726 * *$ & $0.680 * *$ & \\
\hline & $\mathrm{F}$ & & & $0.474^{*}$ & $0.419 *$ & $0.730 * *$ & $0.724 * *$ & $0.693 * *$ & \\
\hline \multirow{3}{*}{$\operatorname{tr}-\mathbf{n}$} & $\mathrm{T}$ & & $0.345^{* *}$ & & & $0.266^{*}$ & $0.328 * *$ & $0.329 * *$ & \\
\hline & M & & $0.688 * *$ & & & & & & \\
\hline & $\mathrm{F}$ & & $0.474 *$ & & & $0.393^{*}$ & & & \\
\hline \multirow{3}{*}{ n-sn } & $\mathrm{T}$ & & & & & & $0.292 *$ & $0.343 * *$ & $0.240 *$ \\
\hline & M & & $0.571 * *$ & & & & & & \\
\hline & $\mathrm{F}$ & & $0.419^{*}$ & & & & & & \\
\hline \multirow{3}{*}{ sn-me } & $\mathrm{T}$ & & $0.321 * *$ & $0.266^{*}$ & & & $0.717 * *$ & $0.737 * *$ & \\
\hline & M & & $0.282 *$ & & & & $0.706^{* *}$ & $0.725^{* *}$ & \\
\hline & $\mathrm{F}$ & & $0.730 * *$ & $0.393 *$ & & & $0.633^{* *}$ & $0.611 * *$ & \\
\hline \multirow{3}{*}{$\begin{array}{c}\text { pn- } \\
\operatorname{pog}(\text { OVD })\end{array}$} & $\mathrm{T}$ & & $0.709 * *$ & $0.328 * *$ & $0.292 *$ & $0.717 * *$ & & $0.952 * *$ & \\
\hline & M & & $0.726 * *$ & & & $0.706^{* *}$ & & $0.958 * *$ & \\
\hline & $\mathrm{F}$ & & $0.724 * *$ & & & $0.633 * *$ & & $0.930 * *$ & \\
\hline \multirow{3}{*}{ pn-pog(RVD) } & $\mathrm{T}$ & $0.356^{* *}$ & $0.725 * *$ & $0.329 * *$ & $0.343 * *$ & $0.737 * *$ & $0.952 * *$ & & \\
\hline & M & & $0.680 * *$ & & & $0.725 * *$ & $0.958 * *$ & & \\
\hline & $\mathrm{F}$ & & $0.693 * *$ & & & $0.611 * *$ & $0.930 * *$ & & \\
\hline \multirow{2}{*}{$\begin{array}{c}\text { Free way } \\
\text { space }\end{array}$} & $\mathrm{T}$ & & & & $0.240^{*}$ & & & & \\
\hline & M & & & & & & & & \\
\hline
\end{tabular}

*Correlation is significant at $\mathrm{p} \leq 0.05$ level; **Correlation is highly significant at $\mathrm{p} \leq 0.01$ level. $\mathrm{OVD}=$ occlusal vertical dimension, $\mathrm{RVD}=$ rest vertical dimension.

In Table (5), the correlation coefficient among all the facial profiles for total sample also reveal different correlation among the variables.
Of most important, is the positive significantly high correlation of pn-pog (OVD) and pn-pog (RVD) with n-me, snme for convex and straight profiles. 
Table (5): The correlation coefficient among all the facial profiles for total sample.

\begin{tabular}{|c|c|c|c|c|c|c|c|c|c|}
\hline Variable & $\begin{array}{l}\text { Facial } \\
\text { pofiles }\end{array}$ & 焉 & $\stackrel{\mathscr{I}}{=}$ & $\stackrel{7}{\leftrightarrows}$ & $\begin{array}{l}0 \\
\stackrel{1}{1} \\
=\end{array}$ & $\underset{\Xi}{\stackrel{\Xi}{E}}$ & 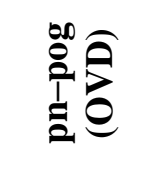 & 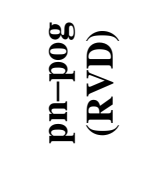 & 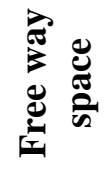 \\
\hline $\begin{array}{l}\text { Soft tissue } \\
\text { facial con- } \\
\text { vexity }\end{array}$ & $\begin{array}{c}\text { Convex } \\
\text { Straight } \\
\text { Concave }\end{array}$ & & & & & & & & \\
\hline n-me & $\begin{array}{l}\text { Convex } \\
\text { Straight } \\
\text { Concave }\end{array}$ & & & $0.399 *$ & $0.646 * *$ & $\begin{array}{l}0.798^{* *} \\
0.837^{* *}\end{array}$ & $\begin{array}{l}0.751 * * \\
0.744 * *\end{array}$ & $\begin{array}{l}0.779 * * \\
0.716^{* *}\end{array}$ & \\
\hline $\operatorname{tr}-\mathbf{n}$ & $\begin{array}{c}\text { Convex } \\
\text { Straight } \\
\text { Concave } \\
\end{array}$ & & $0.399 *$ & & & $0.392 *$ & $0.468 * *$ & $0.450 * *$ & \\
\hline n-sn & $\begin{array}{c}\text { Convex } \\
\text { Straight } \\
\text { Concave } \\
\end{array}$ & & $0.646^{* *}$ & & & $0.331 *$ & $0.450^{*}$ & $0.507 *$ & \\
\hline sn-me & $\begin{array}{c}\text { Convex } \\
\text { Straight } \\
\text { Concave } \\
\end{array}$ & & $\begin{array}{l}0.798 * * \\
0.837 * *\end{array}$ & $0.392 *$ & $0.331 *$ & & $\begin{array}{l}0.794 * * \\
0.689 * *\end{array}$ & $\begin{array}{l}0.825 * * \\
0.691 * *\end{array}$ & \\
\hline $\begin{array}{c}\text { pn- } \\
\operatorname{pog}(\text { OVD })\end{array}$ & $\begin{array}{c}\text { Convex } \\
\text { Straight } \\
\text { Concave }\end{array}$ & & $\begin{array}{l}0.751^{* *} \\
0.744 * *\end{array}$ & $0.468 * *$ & $0.450^{*}$ & $\begin{array}{l}0.794 * * \\
0.689 * *\end{array}$ & & $\begin{array}{l}0.951 * * \\
0.981 * *\end{array}$ & \\
\hline $\begin{array}{c}\text { pn- } \\
\operatorname{pog}(\mathrm{RVD})\end{array}$ & $\begin{array}{c}\text { Convex } \\
\text { Straight } \\
\text { Concave }\end{array}$ & & $\begin{array}{l}0.779 * * \\
0.716^{* *}\end{array}$ & $0.450 * *$ & $0.507 *$ & $\begin{array}{l}0.825^{* *} \\
0.691^{* *}\end{array}$ & $\begin{array}{l}0.951 * * \\
0.981 * *\end{array}$ & & \\
\hline $\begin{array}{c}\text { Free way } \\
\text { space }\end{array}$ & $\begin{array}{c}\text { Convex } \\
\text { Straight } \\
\text { Concave }\end{array}$ & & & & & & & & \\
\hline
\end{tabular}

*Correlation is significant at $p \leq 0.05$ level; ${ }^{* *}$ Correlation is highly significant at $p \leq 0.01$ level. $\mathrm{OVD}=0$ occlusal vertical dimension, $\mathrm{RVD}=$ rest vertical dimension.

\section{DISCUSSION}

In Table (1), soft tissue profile convexity is significantly greater in females indicating tendency of females to have more convex profile than males. The remaining variables are significantly greater in males reflecting sexual dimorphism between sexes. This is in contrast to results of Joson ${ }^{(34)}$ for young adult Filipinos who found that the combined male and female subjects showed a convex profile.

According to subtelny ${ }^{(33)}$, the soft tissue profile grows more convex with age ,despite the tendency of the skeletal profile to straighten out. The profile angle is one measurement that exhibits some gender dimorphism with regard to optimal esthetics. More esthetically pleasing male faces tend toward a straight profile ,but some degree of concavity that comes with a prominent chin is considered attractive. More esthetically pleasing female faces tend toward a mild convexity, with a softer $\operatorname{chin}^{(35)}$.

In this study, in the profile view a general harmony of the forehead, midface and the lower face exists with the lower face height is slightly larger than upper and middle facial heights for total, males and females.

According to Margolis ${ }^{(35)}$, one should focus on the proportions of the face rather than the absolute size. The ideally proportioned face can be divided vertically into equal thirds.

Analysis of variance comparing different facial profiles in Table (2) and Duncan's multiple analysis range test (Table 3 ) 
reveals that $\mathrm{n}-\mathrm{sn}$ and sn-me and free way space significantly differs among the three facial profiles, with concave facial profiles reporting the larger means, while pn-pog (OVD) is significantly smaller in in this face type $(p \leq 0.05)$. This is true since concave face profiles are having long face with increased anterior facial heights. Also this is indicating different growth pattern in these profile types. $\operatorname{tr}-n$, on the other hand, non significantly differs giving clue to the fact that the cranial base developed early and less subjected to effect of other environmental factors that affect growth of facial skeleton.

Arat and Rübendüz ${ }^{(36)}$ in their longitudinal study of changes in dentoalveolar and facial heights during early and late growth periods showed that the differential growth in condylar, sutural and alveolar structures is particularly influential in terms of vertical development of facial characteristics, and alveolar structure plays a compensatory role in establishing sagittal and vertical heights.

The variations in the vertical dimensions are significant when identifying facial types. Therefore, it is important to define the multidimensional combinations in order to make a more accurate identification of the facial types because the interrelation of the anteroposterior and vertical relationship is responsible for the various facial types.

As presented in Table (4), of most important is the correlation of pn-pog (OVD) and pn-pog (RVD) with n-me and sn-me for males, females and total sample, this is true since any increase in total facial height and lower facial height associated with increase in both (OVD) and (RVD). Also is the correlation of free way space with n-sn for total sample.

According to Williams and Wilkin ${ }^{(38)}$, decrease in rest vertical dimension may or may not accompany a decrease in occlusal vertical dimension; it may occur without a decrease in occlusal vertical dimension in patients with a preponderant activity of the jaw-closing musculature, as in patients with muscular hyper tenseness or in chronic gum chewers; increase in rest vertical dimension may or may not accompany an increase in occlusal vertical dimension; it sometimes occurs after the removal of re- maining occlusal contacts, perhaps as a result of the removal of noxious reflex stimuli. However, determination of rest vertical dimension by individual dentists using phonetics and swallowing had wide variations in two of the five patients in a range of up to $6 \mathrm{~mm}^{(39)}$.

In Table (5), the correlation coefficient for the studied variables among all the facial profiles for total sample also reveals important correlation of pn-pog (OVD) and pn-pog (RVD) with n-me, snme for convex and straight profiles. So any increase in total facial height and lower facial height is associated with increase in both (OVD) and (RVD). However, a study have suggested the jaw-muscle spindle as the receptor responsible for regulating and maintaining the occlusal vertical dimension $(\mathrm{OVD})^{(40)}$.

\section{CONCLUSIONS}

From this study, the spatial relationships among various vertical facial heights and the dimensional proportions of different facial profiles in young Iraqi adult students were noticed. The significant differences between males and females for all the studied variables were observed. The different interrelationships among the studied parameters were found which are important parameters that should be understood by the clinician who is planning the appropriate orthodontic therapy for such subjects.

\section{REFERENCES}

1. Joson CA. The Soft Tissue Profile of Selected Young Adult Filipinos with Normal Occlusion. Phil Dent Ass J. 2001; 53(2): 4-10.

2. Maidl MM, Evans CA, Viana G, Anderson NK, Giddon DB. Preferences for Facial Profiles Between Mexican Americans and Caucasians. Angle Orthod. 2005; 75(6): 953-958.

3. Giddon DB. Orthodontic applications of psychological and perceptual studies of facial esthetics. Semin Orthod. 1995; 1: 82-93.

4. Wells KB, Golding JM, Hough RL, Burnam A, Karno M. Factors affecting the probability of use of general and medical health and social/community services for Mexican Americans and non-Hispanic 
whites. Med Care. 1988; 26: 441-452.

5. Rakosi TH. An atlas and manual of cephalometric radiography. $2^{\text {nd }}$ ed. Wolfe Medical Puplications Ltd. Great Britain. 1982; Pp: 80-83.

6. Ra'uf FMS. Facial analysis and facial types of the students in Mosul University, normal occlusion. The direct method. MSc Thesis. College of Dentistry. University of Mosul. 1997.

7. Bos A, Hoogstraten J, Prahl-Andersen A. Expectations of treatment and satisfaction with dentofacial appearance in orthodontic patients. Am J Orthod Dentofacial Orthop. 2003; 123(2): 127-132.

8. Bishara SE, Jorgensen GJ, Jakobsen JR. Changes in facial dimensions assessed from lateral and frontal photographs. Part I-Methodology. Am J Orthod Dentofacial Orthop. 1995; 108(4): 389-393.

9. Ramadan OZ. Relation between photographic facial measurements and lower dental arch measurements in adult Jourdanian males with Class I normal occlusion. MSc Thesis. College of Dentistry. University of Mosul. 2000.

10.Bishara SE, Jacobsen JR. Soft tissue profile changes from 5 to 45 years of age. Am J Orthod Dentofacial Orthop. 1998; 114(6): 698-706.

11.Agha NF. Facial profile soft tissue analysis for Mosuli adults, Class I normal occlusion (a cephalometric study). MSc Thesis. College of Dentistry. University of Mosul. 1998.

12. Anthropometry. from Wikipedia, the free encyclopedia. Retrieved from HYPERLINK http://en.wikipedia.org/wiki/ Anthropometry

"/http://en.wikipedia.org/wiki/Anthropome try" This paper was last modified on 24 Aug 2007.

13. Werli T, Matis R, Hedelin G, RotheaGouillard C. Aesthetic assessment of soft tissue profile by visual art students. Revue d'Orthopedie Dento Faciale. 2003; 24(5): 145-156.

14.Proffit WR, Fields HW, Ackerman JL, Sinclair PM, Thomas PM, Tulloch JF. Contemporary Orthodontics. $3^{\text {rd }}$ Ed. Mosby. St Louis. 2000; Pp: 16-94.

15. Coward TJ, Scott BJ, Watson RM, Richards R. Laser scanning of the ear identifying the shape and position in subjects with normal facial asymmetry. Int J Oral
Maxillofac Surg. 2000; 18: 23-29.

16.Douglas TS, Martinez F, Meintjes EM, Vaughan CL, Viljoen DL. Eye feature extraction for diagnosing the facial phenotype associated with fetal alcohol syndrome. Med Biol Eng Comput. 2003; 41:101-106.

17. Duffy S, Noar JH, Evans RD, Sanders R. Three-dimensional analysis of the child cleft face. Cleft Palate Craniofac J. 2000; 37:137-144.

18. Ferrario VF, Dellavia C, Tartaglia GM, Turci M, Sforza C. Soft tissue facial morphology in obese adolescents: a threedimensional noninvasive assessment. Angle Orthod. 2004; 74(1): 37-42.

19. Meintjes EM, Douglas TS, Martinez F, Vaughan CL, Adams LP, Stekhoven A, Viljoen D. A stereo-photogrammetric method to measure the facial dysmorphology of children in the diagnosis of fetal alcohol syndrome. Med Eng Phys. 2002; 24: 683689.

20. Shaner DJ, Peterson AE, Beattie OB, Bamforth JS. Assessment of soft tissue facial asymmetry in medically normal and syndrome-affected individuals by analysis of landmarks and measurements. Am J Med Genet. 2000; 93: 143-154.

21. Chitkara U, Lee L, Oehlert JW, Bloch DA, Holbrook RHJr, El-Sayed YY, Druzin ML. Fetal ear length measurement: a useful predictor of aneuploidy? Ultrasound Obstet Gynecol. 2002; 19: 131-135.

22. Sepulveda W, Sandoval R, Carstens E, Gutierrez J, Vasquez P. Hypohidrotic ectodermal dysplasia: prenatal diagnosis by three-dimensional ultrasonography. $\mathrm{J} \mathrm{Ul}$ trasound Med. 2003; 22: 731-735.

23. Ferrario VF, Sforza C, Serrao G, Miani AJr. A computerized method for the assessment of human facial volume. J Craniomaxillofac Surg. 1995; 23: 280-286.

24.Fayz F, Eslami A. Determination of occlusal vertical dimension: literature review. J Prosth Dent. 1988; 59: 321-323.

25.Bloom DR, Padayachy JN. Increasing occlusal vertical dimension-why, when and how? Brit Dent J. 2006; 200(4): 199203.

26. Heart well CM, Rahn AO. Syllabus of complete denture. $4^{\text {th }}$ ed. Philadelphia. Lea and Febiger. 1986; Pp: 228-230.

27.Jones D, Hill K. Criteria of facial attractiveness in five populations. Hum Nat. 
1993; 4: 271-295.

28. Kim JY, Lee SJ, Kim TW, Nahm DS, Chang YI. Classification of skeletal variation in normal occlusion. Angle Orthod. 2004; 75 (3): 303-311.

29.Zylinski CG, Nanda RS, Kapila S. Soft tissue profile in white males. Am J Orthod Dentofacial Orthop. 1992; 101(6): 514518.

30. Robert LE. Complete denture esthetic. Dent Clinic North Am. 1996; 40(1): 71.

31.Douglas $\mathrm{Du}$. Changes in the Caucasian male facial profile as depicted in fashion magazines during thetwentieth century. Am J Orthod Dentofacial Orthop. 1998; 114(8): 208-217.

32. No AUTHORS. Glossary of prosthodontic terms. J Prosth Dent. 2005; 94(1): 5761.

33. Subtelny JD. A longitudinal study of soft tissue profile of the Negro population. Am J Orthod. 1959; 45(7): 481-507.

34.Joson CA. The soft tissue profile of selected young adult Filipinos with normal occlusion. Philipp Dent Assoc J. 2001; 53: 24-10.
35. Margolis MJ. Esthetic considerations in orthodontic trearment of adults. Adult orthodontics II. Dent Clinic North Am. 1997; 41(1): 29-48.

36. Arat $\mathrm{Z}$ M, Rübendüz M. Changes in dentoalveolar and fracial heights during early and late growth periods. A Longitudinal Study. Angle Orthod. 2004; 75(1): 69-74.

37. Kim J, Lee S, Kim T, Nahm D, Chang Y. Classification of the skeletal variation in normal occlusion. Angle Orthod. 2004; 75 ( 3): 303-311.

38. Williams L, Wilkin S. Stedman's medical dictionary. $26^{\text {th }}$ ed. Copyright $(9)$ 2006. P: 213.

39. Feldman S, Leupold RJ, Staling LM. Rest vertical dimension determined by electromyography with biofeedback as compared to conventional methods. J Prosthet Dent. 1978; 40(2): 216-219.

40. Yabushita T, Zeredo JL, Toda K, Soma K. Role of occlusal vertical dimension in spindle function. J Dent Res. 2005; 84(3): 245-249. 